\title{
AN APPLICATION OF SOME FRACTIONAL INTEGRALS
}

\author{
by J. S. LOWNDES
}

(Received 7 July, 1977)

1. A discussion of the Erdélyi-Kober operator

$$
I_{\eta, \alpha} f(x)=\frac{2 x^{-2(\eta+\alpha)}}{\Gamma(\alpha)} \int_{0}^{x}\left(x^{2}-u^{2}\right)^{\alpha-1} u^{2 \eta+1} f(u) d u \quad(\alpha>0)
$$

and its adjoint operator $K_{n, \alpha}$ together with an account of some of their applications can be found in the book by Sneddon [5].

Erdélyi [1] has shown that this operator can be used to connect singular differential operators of the form

$$
L_{\lambda}=x^{-(2 \lambda+1)} \frac{d}{d x} x^{2 \lambda+1} \frac{d}{d x} .
$$

He proved, under appropriate conditions, that

$$
I_{\eta, \alpha} L_{\eta} f(x)=L_{\eta+\alpha} I_{\eta, \alpha} f(x)
$$

and demonstrated that this relation could be used in work on partial differential equations involving the operator $L_{\lambda}$ which occur in generalized axially symmetric potential theory. A comprehensive list of references to this and related topics is given in Gilbert's book [2]

In this paper we consider the generalized Erdélyi-Kober operators [4] defined by

$$
\Im_{k}(\eta, \alpha) f(x)=2^{\alpha} x^{-2(\eta+\alpha)} k^{1-\alpha} \int_{0}^{x} u^{2 \eta+1}\left(x^{2}-u^{2}\right)^{(\alpha-1) / 2} J_{\alpha-1}\left\{k \sqrt{ }\left(x^{2}-u^{2}\right)\right\} f(u) d u,
$$

where $\alpha>0, k \geqslant 0, J_{\alpha-1}(x)$ is the Bessel function of the first kind and the operator $\Im_{i k}(\eta, \alpha)$, which is defined as in equation (4) but with $J_{\alpha-1}$ replaced by $I_{\alpha-1}$, the modified Bessel function of the first kind.

These operators have the properties

$$
\begin{gathered}
\Im_{k}(\eta, \alpha) x^{2 \beta} f(x)=x^{2 \beta} \Im_{k}(\eta+\beta, \alpha) f(x), \Im_{k}(\eta+\alpha, \beta) \Im_{i k}(\eta, \alpha)=I_{\eta, \alpha+\beta}, \\
\Im_{k}^{-1}(\eta, \alpha)=\Im_{i k}(\eta+\alpha,-\alpha), \Im_{i k}^{-1}(\eta, \alpha)=\Im_{k}(\eta+\alpha,-\alpha) .
\end{gathered}
$$

Defining the adjoint operator $\Re_{k}(\eta, \alpha)$ by

$$
\int_{0}^{\infty} x f(x) \Im_{k}(\eta, \alpha) g(x) d x=\int_{0}^{\infty} x g(x) \mathfrak{A}_{k}(\eta, \alpha) f(x) d x
$$

we find that it is given by the formula [4]

$$
\Re_{k}(\eta, \alpha) f(x)=2^{\alpha} x^{2 \eta} k^{1-\alpha} \int_{x}^{\infty} u^{1-2 \eta-2 \alpha}\left(u^{2}-x^{2}\right)^{(\alpha-1) / 2} J_{\alpha-1}\left\{k \sqrt{ }\left(u^{2}-x^{2}\right)\right\} f(u) d u,
$$

when $\alpha>0$.

Glasgow Math. J. 20 (1979) 35-41. 
Under stated assumptions we first of all prove that

$$
\Im_{k}(\eta, \alpha) L_{\eta} f(x)=\left(L_{\eta+\alpha}+k^{2}\right) \Im_{k}(\eta, \alpha) f(x),
$$

which reduces to equation (3) when $k=0$ since $\Im_{0}(\eta, \alpha)=I_{\eta, \alpha}$.

This result is then applied to deduce properties of the solutions of the generalized axially symmetric Helmholtz equation from the corresponding solutions of Laplace's equation in two-dimensions.

No claim about the originality of the results presented in this paper is made, but it is felt that the elementary use of operators of fractional integration to obtain them might appeal to the applied mathematician.

2. We shall now prove the following result.

(i) If $\alpha>0, f \in C^{2}(0, b)$ for some $b>0, x^{2 \eta+1} f(x)$ is integrable at the origin and $x^{2 n+1} f^{\prime}(x) \rightarrow 0$ as $x \rightarrow 0$; or

(ii) if $\alpha<0, H=x^{2(\eta+\alpha)} \Im_{i k}(\eta, \alpha) f(x)$ exists, $H \in C^{2}(0, b), x H(x)$ is integrable at the origin and

$$
x H^{\prime}(x)-2(\eta+\alpha) H(x) \rightarrow 0 \quad \text { as } x \rightarrow 0
$$

then

$$
\Im_{k}(\eta, \alpha) L_{n} f(x)=\left(L_{\eta+\alpha}+k^{2}\right) \Im_{k}(\eta, \alpha) f(x)
$$

Proof of (i)

We set

$$
\begin{aligned}
G(x) & =\Im_{k}(\eta, \alpha) f(x) \\
& =2^{\alpha}(k x)^{1-\alpha} \int_{0}^{1} t^{1+2 \eta}\left(1-t^{2}\right)^{(\alpha-1) / 2} J_{\alpha-1}(\xi) f(x t) d t,
\end{aligned}
$$

where $\alpha>0$ and $\xi=k x \sqrt{ }\left(1-t^{2}\right)$.

Now $G(x)$ is differentiable and we find that

$$
\begin{aligned}
G^{\prime}(x)= & -2^{\alpha} k(k x)^{1-\alpha} \int_{0}^{1} t^{1+2 \eta}\left(1-t^{2}\right)^{\alpha / 2} J_{\alpha}(\xi) f(x t) d t \\
& +2^{\alpha}(k x)^{1-\alpha} \int_{0}^{1} t^{2+2 \eta}\left(1-t^{2}\right)^{(\alpha-1) / 2} J_{\alpha-1}(\xi) f^{\prime}(x t) d t .
\end{aligned}
$$

Integrating the second integral in this equation by parts we see that with our assumptions the integrated part vanishes and equation (12) becomes

$$
G^{\prime}(x)=2^{\alpha} x(k x)^{-\alpha} \int_{0}^{1} t^{1+2 \eta}\left(1-t^{2}\right)^{\alpha / 2} J_{\alpha}(\xi)\left[F(x t)-k^{2} f(x t)\right] d t,
$$


where we have written

$$
F(x)=x^{-(2 \eta+1)} \frac{d}{d x} x^{2 \eta+1} f^{\prime}(x) .
$$

On applying the operator $x^{-(2 n+1)}(d / d x) x^{2 \eta+1}$ to equation (12) it can easily be shown that we get

$$
\begin{aligned}
L_{\eta} G(x)= & 2^{\alpha} k^{2}(k x)^{-\alpha} \int_{0}^{1} t^{1+2 \eta}\left(1-t^{2}\right)^{\alpha / 2}\left[\xi J_{\alpha+1}(\xi)-2(1+\eta) J_{\alpha}(\xi)\right] f(x t) d t \\
& +2^{\alpha}(k x)^{1-\alpha} \int_{0}^{1} t^{3+2 \eta}\left(1-t^{2}\right)^{(\alpha-1) / 2} J_{\alpha-1}(\xi) F(x t) d t \\
& -2^{\alpha+1} k(k x)^{1-\alpha} \int_{0}^{1} t^{2+2 \eta}\left(1-t^{2}\right)^{\alpha / 2} J_{\alpha}(\xi) f^{\prime}(x t) d t
\end{aligned}
$$

Integrating the last integral in the above equation by parts we find that the integrated part vanishes and it becomes

$$
-2^{\alpha+1}(k x)^{1-\alpha} \int_{0}^{1} t^{1+2 \eta}\left(1-t^{2}\right)^{(\alpha+1) / 2} J_{\alpha+1}(\xi) F(x t) d t .
$$

Combining equations (11), (13), (15) and (16) we obtain

$$
\begin{array}{r}
\left(L_{\eta+\alpha}+k^{2}\right) G(x)=\Im_{k}(\eta, \alpha) L_{\eta} f(x) \\
+2^{\alpha}\left(\frac{k}{x}\right)(k x)^{-\alpha} \int_{0}^{1} t^{1+2 \eta}\left(1-t^{2}\right)^{(\alpha-1) / 2}\left[(k x t)^{2} J_{\alpha-1}(\xi)-2(1+\eta) \xi J_{\alpha}(\xi)\right] f(x t) d t \\
\quad-2^{\alpha}(k x)^{1-\alpha} \int_{0}^{1} t^{1+2 \eta}\left(1-t^{2}\right)^{(\alpha+1) / 2} J_{\alpha+1}(\xi) F(x t) d t .
\end{array}
$$

Finally, performing an integration by parts twice on the last integral in equation (17) and noting that under our assumptions the integrated parts vanish, it can be shown that the second and third terms on the right hand side of the equation cancel and we are left with the required result given by equation (10).

In a similar way we can prove that

$$
\Im_{i k}(\eta, \alpha) L_{\eta} f(x)=\left(L_{\eta+\alpha}-k^{2}\right) \Im_{i k}(\eta, \alpha) f(x) .
$$

Proof of (ii)

Here we write

$$
G_{1}(x)=\Im_{i k}(\eta, \alpha) f(x)=x^{-2(\eta+\alpha)} H(x)
$$

and using the results (6) we see that equation (18) may be written as

$$
\Im_{k}(\eta+\alpha,-\alpha) L_{\eta+\alpha} G_{1}(x)=\left(L_{\eta}+k^{2}\right) \Im_{k}(\eta+\alpha,-\alpha) G_{1}(x) .
$$

But $-\alpha>0$ and we can apply to $G_{1}(x)$ the result already proved in (i), with $\eta$ replaced by 
$\eta+\alpha$. In this case $G_{1} \in C^{2}(0, b), x^{2(\eta+\alpha)+1} G_{1}(x)=x H(x)$ is integrable at the origin and

$$
x^{2(\eta+\alpha)+1} G_{1}^{\prime}(x)=x H^{\prime}(x)-2(\eta+\alpha) H(x) \rightarrow 0
$$

as $x \rightarrow 0$. This completes the proof of (ii).

3. The operator $L_{\lambda}$ appears in many partial differential equations [2], for example

$$
M_{\eta} u(x, y)=\frac{\partial^{2} u}{\partial x^{2}}+\frac{(2 \eta+1)}{x} \frac{\partial u}{\partial x}+\frac{\partial^{2} u}{\partial y^{2}}=0
$$

which is the equation satisfied by axially symmetric solutions of Laplace's equation in $(2 \eta+3)$-dimensions. In general $\eta$ need not be an integer and equation (20) is called the generalized axially symmetric potential equation (GASPE), a study of which has given rise to generalized axially symmetric potential theory (GASPT).

As a consequence of the result $(10)$ we have that

$$
\Im_{k}(\eta, \alpha) M_{\eta} u(x, y)=\left(M_{n+\alpha}+k^{2}\right) \Im_{k}(\eta, \alpha) u(x, y),
$$

under appropriate conditions on $u(x, y)$.

The equation

$$
\left(M_{n+\alpha}+k^{2}\right) v(x, y)=0
$$

is known as the generalized axially symmetric Helmholtz equation (GASHE) in ( $2 \eta+$ $2 \alpha+3)$ dimensions [2]. result.

Following reasoning similar to that used by Erdélyi [1] we can deduce the following

If $\eta>-1, \eta+\alpha>-1$ and $u(x, y)$ is a solution of the $(2 \eta+3)$ dimensional GASPE then $\Im_{k}(\eta, \alpha) u(x, y)$ exists and is a solution of the $(2 \eta+2 \alpha+3)$ dimensional GASHE. equation

4. As a first example let $h(x, y)$ be a solution of the two dimensional Laplace's

$$
\frac{\partial^{2} h}{\partial x^{2}}+\frac{\partial^{2} h}{\partial y^{2}}=0
$$

which satisfies the condition $h_{x}(0, y)=0$; i.e. $h$ is an even function of $x$.

Then if $\alpha>-1$,

$$
u(x, y)=\Im_{k}\left(-\frac{1}{2}, \alpha+\frac{1}{2}\right) h(x, y)
$$

exists and is a solution of the $(2 \alpha+3)$ dimensional GASHE

$$
\left(M_{\alpha}+k^{2}\right) u(x, y)=0 .
$$

In order to find an explicit solution of the above equation subject to the boundary 
condition $u(0, y)=g(y)$ we have from equation (24) that

$$
\begin{aligned}
g(y) & =u(0, y) \\
& =\lim _{x \rightarrow 0} \Im_{k}\left(-\frac{1}{2}, \alpha+\frac{1}{2}\right) h(x, y)=\frac{\Gamma\left(\frac{1}{2}\right)}{\Gamma(\alpha+1)} h(0, y),
\end{aligned}
$$

which shows that the corresponding even harmonic function is given by

$$
h(x, y)=\frac{\Gamma(\alpha+1)}{2 \Gamma\left(\frac{1}{2}\right)}[g(y+i x)+g(y-i x)] .
$$

From equation (24) we see that the solution of equation (25) which satisfies the condition $u(0, y)=g(y)$ is

$$
u(x, y)=\frac{\Gamma(\alpha+1)}{2 \Gamma\left(\frac{1}{2}\right)} \Im_{k}\left(-\frac{1}{2}, \alpha+\frac{1}{2}\right)[g(y+i x)+g(y-i x)]
$$

provided that $g(s+i t)$ is analytic for $s=y,-x \leqslant t \leqslant x$.

When $\alpha>-\frac{1}{2}$, we have, on using the definition (4), that

$$
\begin{aligned}
u(x, y)= & \frac{2^{\alpha-1 / 2} \Gamma(\alpha+1)}{x^{2 \alpha} \Gamma\left(\frac{1}{2}\right)} k^{1 / 2-\alpha} \int_{0}^{x}\left(x^{2}-t^{2}\right)^{(\alpha-1 / 2) / 2} J_{\alpha-1 / 2}\left\{k \sqrt{ }\left(x^{2}-t^{2}\right)\right\} \\
& \times[g(y+i t)+g(y-i t)] d t,
\end{aligned}
$$

from which we obtain the following expression for $u(x, y)$ in terms of its axial value $u(0, y)$.

$$
u(x, y)=\frac{\Gamma(\alpha+1)}{\Gamma\left(\frac{1}{2}\right)}\left(\frac{k x}{2}\right)^{1 / 2-\alpha} \int_{0}^{\pi}(\sin \theta)^{\alpha+1 / 2} J_{\alpha-1 / 2}(k x \sin \theta) u(0, y+i x \cos \theta) d \theta
$$

This agrees with the result found by Henrici [3] (cf. the representation given by Gilbert [2, p. 214]). Letting $k \rightarrow 0$ in the above equation we obtain the Laplace integral representation of solutions of the $(2 \alpha+3)$ dimensional GASPE given by Erdélyi [1].

5. For a second example consider the two dimensional Laplace's equation (23) with the boundary conditions $h(x, 0)=f(x), 0<x<\infty, h_{x}(0, y)=0,0<y<\infty$ and $h \rightarrow 0$ as $y \rightarrow \infty$.

The solution of this problem can be written as

$$
h(x, y)=\frac{2}{\pi} \int_{0}^{\infty} A(\xi) e^{-\xi y} \cos (\xi x) d \xi
$$

where

$$
A(\xi)=\int_{0}^{\infty} f(t) \cos (t \xi) d t
$$


Using the previous results we find that the solution of the $(2 \alpha+3)$ dimensional GASHE

$$
\frac{\partial^{2} u}{\partial x^{2}}+\frac{(2 \alpha+1)}{x} \frac{\partial u}{\partial x}+\frac{\partial^{2} u}{\partial y^{2}}+k^{2} u=0 \quad(0<x, y<\infty),
$$

where $\alpha>-\frac{1}{2}, u(x, 0)=\mathfrak{I}_{k}\left(-\frac{1}{2}, \alpha+\frac{1}{2}\right) f(x)$ and $u \rightarrow 0$ as $y \rightarrow \infty$, is given by

$$
\begin{aligned}
u(x, y) & =\Im_{k}\left(-\frac{1}{2}, \alpha+\frac{1}{2}\right) h(x, y) \\
& =\frac{2}{\pi} \int_{0}^{\infty} A(\xi) e^{-\xi y} \Im_{k}\left(-\frac{1}{2}, \alpha+\frac{1}{2}\right) \cos (\xi x) d \xi \\
& =\frac{2^{\alpha+1} x^{-\alpha}}{\sqrt{\pi}} \int_{0}^{\infty} \frac{A(\xi) e^{-\xi y}}{\left(\xi^{2}+k^{2}\right)^{\alpha / 2}} J_{\alpha}\left\{x \sqrt{ }\left(\xi^{2}+k^{2}\right)\right\} d \xi,
\end{aligned}
$$

where $A(\xi)$ is defined by equation (32) and $\Im_{k}\left(-\frac{1}{2}, \alpha+\frac{1}{2}\right) \cos (\xi x)$ has been evaluated using a result in [5, p. 31].

Confining our attention to the three dimensional case $(\alpha=0)$, we have that the usual form of the solution of the Helmholtz equation subject to the conditions given above is

$$
u_{3}(x, y)=\int_{0}^{\infty} \lambda B(\lambda) e^{-y \sqrt{ }\left(\lambda^{2}-k^{2}\right)} J_{0}(\lambda x) d \lambda,
$$

where $\sqrt{ }\left(\lambda^{2}-k^{2}\right)=-i \sqrt{ }\left(k^{2}-\lambda^{2}\right), k>\lambda$, and

$$
B(\lambda)=\int_{0}^{\infty} t J_{0}(\lambda t) \Im_{k}\left(-\frac{1}{2}, \frac{1}{2}\right) f(t) d t
$$

Using the result (7), equation (36) can be written as

$$
\begin{aligned}
B(\lambda) & =\int_{0}^{\infty} t f(t) \Re_{k}\left(-\frac{1}{2}, \frac{1}{2}\right) J_{0}(\lambda t) d t \\
& =\frac{2 H(\lambda-k)}{\sqrt{\left[\pi\left(\lambda^{2}-k^{2}\right)\right]}} \int_{0}^{\infty} f(t) \cos \left[t \sqrt{ }\left(\lambda^{2}-k^{2}\right)\right] d t,
\end{aligned}
$$

where $H(x)$ is the Heaviside unit function and $\Re_{k}\left(-\frac{1}{2}, \frac{1}{2}\right) J_{0}(\lambda t)$ has been evaluated using a result in $[5$, p. 31].

After an obvious change of variable it is easily seen that the solution given by equations (35) and (37) agrees with that given by equations (34) and (32) when $\alpha=0$.

\section{REFERENCES}

1. A. Erdélyi, An application of fractional integrals, J. Analyse Math. 14 (1965), 113-126. 1969).

2. R. P. Gilbert, Function theoretic methods in partial differential equations (Academic Press,

3. P. Henrici, Complete systems of solutions for a class of singular elliptic partial differential equations, Boundary problems in differential equations (University of Wisconsin Press, Wisconsin, 1960), 19-34. 
4. J. S. Lowndes, A generalisation of the Erdélyi-Kober operators, Proc. Edinburgh Math. Soc. $17(1970), 139-148$.

5. I. N. Sneddon, Mixed boundary value problems in potential theory (North Holland, 1966).

Department of Mathematics

UNIVERSITY OF STRATHCLYDE

GlasGow. 